\title{
Transcatheter Device Closure of a Perimembranous Ventricular Septal Defect in Congenitally Corrected Transposition of the Great Arteries
}

\author{
Catherine E. Tomasulo ${ }^{1, *}$, Lindsay S. Rogers ${ }^{1}$, Lauren Andrade ${ }^{1,2}$ and Michael L. O’Byrne ${ }^{1,3,4}$ \\ ${ }^{1}$ Division of Cardiology, The Children's Hospital of Philadelphia, Philadelphia, USA \\ ${ }^{2}$ Division of Cardiology, Hospital of the University of Pennsylvania, Philadelphia, USA \\ ${ }^{3}$ Center for Pediatric Clinical Effectiveness, The Children's Hospital of Philadelphia, Philadelphia, USA \\ ${ }^{4}$ Leonard Davis Institute and Center for Cardiovascular Outcomes, Quality, and Evaluative Research, University of Pennsylvania, \\ Philadelphia, USA \\ *Corresponding Author: Catherine E. Tomasulo. Email: caelto@gmail.com
}

Received: 01 June 2021 Accepted: 10 August 2021

\begin{abstract}
The majority of patients with congenitally corrected transposition of the great arteries, also known as transposition of the great arteries $\{\mathrm{S}, \mathrm{L}, \mathrm{L}\}$ have ventricular septal defects (VSD), most commonly perimembranous VSD (pmVSD). Transcatheter device closure of pmVSD in these patients has not been widely described. We present a case of device closure of pmVSD in L-TGA with an Amplatzer Duct Occluder II (ADOII) device using a deployment starting in the subpulmonary left ventricle. The case demonstrates some of the technical advantages of the ADOII device for VSD closure, specifically its low profile, symmetric shape, and soft material. These characteristics are advantageous in closing conventional pmVSD but are especially useful in patients with challenging anatomic substrates.
\end{abstract}

\section{KEYWORDS}

Percutaneous; catheterization; congenital heart disease; thromboembolism

\section{Introduction}

Congenitally corrected transposition of the great arteries, known as transposition of the great arteries $\{\mathrm{S}, \mathrm{L}, \mathrm{L}\}$ (L-TGA) is frequently associated with additional cardiac defects: ventricular septal defects, specifically perimembranous ventricular septal defects (pmVSD) in series outside of Asia (60-80\%), pulmonary outflow tract obstruction (30-50\%), and abnormalities of the tricuspid valve $(90 \%$ prevalence on pathology though less frequently significant in vivo) [1]. Transcatheter device closure of pmVSD has become more widely utilized in general; however, it is complicated in L-TGA due to several factors, including (1) location of the VSD in L-TGA is closer to the pulmonary valve and more remote from the aortic valve compared to D-looped ventricles with normally related great arteries, (2) crossing the defect retrograde (i.e., from systemic to pulmonary ventricle) is challenging because of sub-aortic right ventricular trabeculations, and (3) the tricuspid valve is frequently abnormal in L-TGA. These structural and functional abnormalities can potentially complicate seating a device in the sac created by aneurysmal 
tricuspid valve tissue. In addition, abnormal conduction tissue in L-TGA is associated with an increased likelihood of heart block (both spontaneously and complicating open heart surgery and catheterizations).

We present a case of transcatheter closure of a pmVSD with an Amplatzer Duct Occluder II (ADOII) (St. Jude Medical, St. Paul, MN, USA) in a patient with L-TGA. The case is illustrative of the potential advantages of using the ADOII for transcatheter VSD closure not only in the specific case of L-TGA but also in the more common situation of a pmVSD in a patient with D-looped ventricles.

\section{Case Report}

The patient is a 21-year-old young man born with L-transposition of the great arteries $\{\mathrm{S}, \mathrm{L}, \mathrm{L}\}$ with a moderate pmVSD with pulmonary stenosis and a competent tricuspid valve. The resulting physiology allowed for relatively normal oxygen saturations and exercise tolerance. As a result, he had never undergone operative intervention for his anatomic heart disease and was followed conservatively by his local cardiologist. At 14 years of age, he developed a high-grade atrioventricular block and underwent placement of a transvenous dual-chamber pacemaker. His pmVSD was not addressed at the time, with a catheterization demonstrating a single pressure-restrictive defect with a modest shunt.

The patient came to our attention because of a progressive increase in the pacing threshold of his ventricular lead, for which he was referred to specialists in adult congenital heart disease and cardiac electrophysiology at our institution. Though the VSD was pressure restrictive and not thought to be a source of a hemodynamically significant shunt in either direction, the electrophysiologist was concerned that the presence of a shunt placed him at risk for thromboembolic stroke both acutely during planned percutaneous lead extraction and replacement, as well as in the indefinite future. Stroke due to paradoxical embolus has been described after transvenous pacemaker placement in the setting of ventricular inversion with a VSD [2]. Epicardial lead placement, initiation of anticoagulation, and/or operative closure of the VSD were also discussed. Surgical VSD closure was not indicated from the perspective of addressing a significant left-to-right shunt. Epicardial lead placement would not address the patient's indwelling leads and risk for thromboembolism. Another advantage of transcatheter closure of VSD would be to avoid lifelong anticoagulation, which would be recommended by the electrophysiologist if the transvenous pacemaker was replaced and the VSD remained open. As a result (and in light of the patient's preference to avoid open-heart surgery), transcatheter device closure of the VSD with transesophageal echocardiography (TEE)-guidance was planned.

The patient was brought to the catheterization laboratory, where he underwent a hemodynamic evaluation and TEE. TEE demonstrated a single pmVSD partially covered by accessory tricuspid valve tissue billowing into the sub-pulmonary left ventricle (LV), with a single orifice in the aneurysmal tricuspid valve tissue that was $5 \mathrm{~mm}$ in diameter (Fig. 1). As expected, the VSD was remote from the aortic valve. The hemodynamic evaluation demonstrated a small magnitude left-to-right shunt (ratio of pulmonary to systemic blood flow of 1.4:1), normal pulmonary artery pressures and mildly elevated leftsided filling pressures (sub-aortic right ventricular end-diastolic pressure was $15 \mathrm{mmHg}$ ). Angiography was consistent with the TEE measurement (Fig. 1a).

Our typical approach to closing pmVSD is similar to that described in other series [3]. Typically, the VSD is engaged with a Judkins right coronary catheter, and an 0.035 inch J-tipped Noodle Wire (AGA Medical, Golden Valley, MN, USA) is advanced across the defect from systemic to the pulmonary ventricle. The wire is snared and externalized to create a veno-arterial loop over which the closure device is deployed antegrade (i.e., with the distal disc in the LV). 

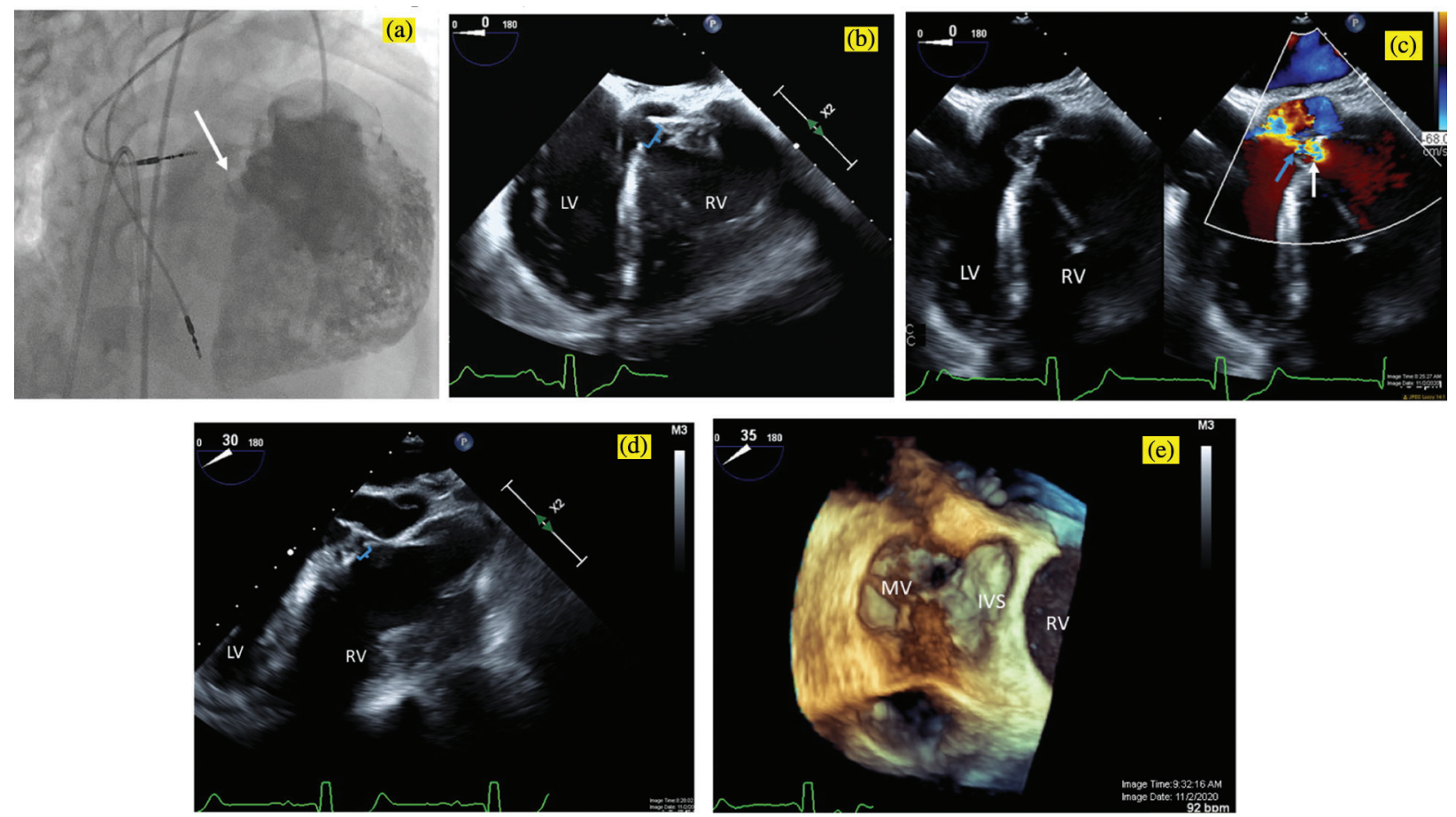

Figure 1: Pre-intervention imaging. A power injection angiogram in an $\mathrm{RAO} / \mathrm{Cranial}$ projection (a) demonstrates a single perimembranous VSD (white arrow), which is remote from the aortic valve and is partially covered by accessory tricuspid valve tissue, with a single orifice in the aneurysmal tricuspid valve tissue measuring $5 \mathrm{~mm}$ in diameter. Transesophageal echocardiogram in a zero-degree view similarly (b) demonstrates the $5 \mathrm{~mm}$ ventricular septal defect (brackets) with accessory tricuspid valve tissue partially covering the defect. With color compare (c), the VSD is seen in 2D on the left, and with the color flow on the right, aliasing can be seen beginning at anatomic VSD (white arrow) and continuing through the restrictive aneurysmal tricuspid valve tissue (blue arrow). Transesophageal echocardiogram in a 30-degree view (d) demonstrates the proximity of the VSD (brackets) to the aortic valve and in a 35degree view with 3D Zoom acquisition (e) shows the VSD from the LV apex view with the anterior leaflet of the mitral valve on the left (MV). IVS, interventricular septum; LV, left ventricle; RV, right ventricle; VSD, ventricular septal defect

For a number of reasons, this approach was challenging in this patient. The VSD was remote from the aortic valve and could not be engaged using a Judkins coronary catheter. Instead, an Amplatz left 1 (AL-1) catheter (Cook Medical, Bloomington, IN, USA) was used to engage the defect under fluoroscopic guidance and confirmed on TEE. The floppy J-tip of the wire would not pass cleanly. The wire buckled and forced the catheter out of position even when the VSD was cleanly engaged on fluoroscopy and TEE. Instead, a 0.035 inch angle-tipped hydrophilic glide wire (Terumo Corp, Elkton, MD, USA) was selected and directed across the defect without incident (Fig. 2a). The AL-1 catheter tracked over this wire into the subpulmonary LV. Rather than forming a veno-arterial loop, we decided to utilize a retrograde deployment of an ADOII device. To do this, we exchanged the catheter and wire for a 0.035 inch Rosen guidewire (Cook Medical, Bloomington, IN, USA), and with some manipulation of the coronary catheter, we were able to achieve a redundant loop of wire in the LV cavity (Fig. 2b). A TorqVue delivery system tracked retrograde over the wire and through the defect without issue (Fig. 2c). Under a combination of fluoroscopic and TEE guidance, the device was unsheathed and then deployed. Initially, the device would not pull back cleanly to appose the septum, which we determined was due to it being trapped in the 
mitral valve apparatus. The device was re-sheathed without difficulty, and the left ventricular disc was opened closer to the septum. The partially unsheathed device and delivery system were pulled back as a unit without difficulty until flush against the septal wall. The rest of the device was unsheathed and appeared to be in a good position vis the septum (Fig. 2d). There was no hemodynamic derangement, and the patient remained in his usual paced rhythm without issue. After TEE and fluoroscopic inspection, the device was released. It reoriented once released from its delivery cable but remained in a good position (Fig. 3).
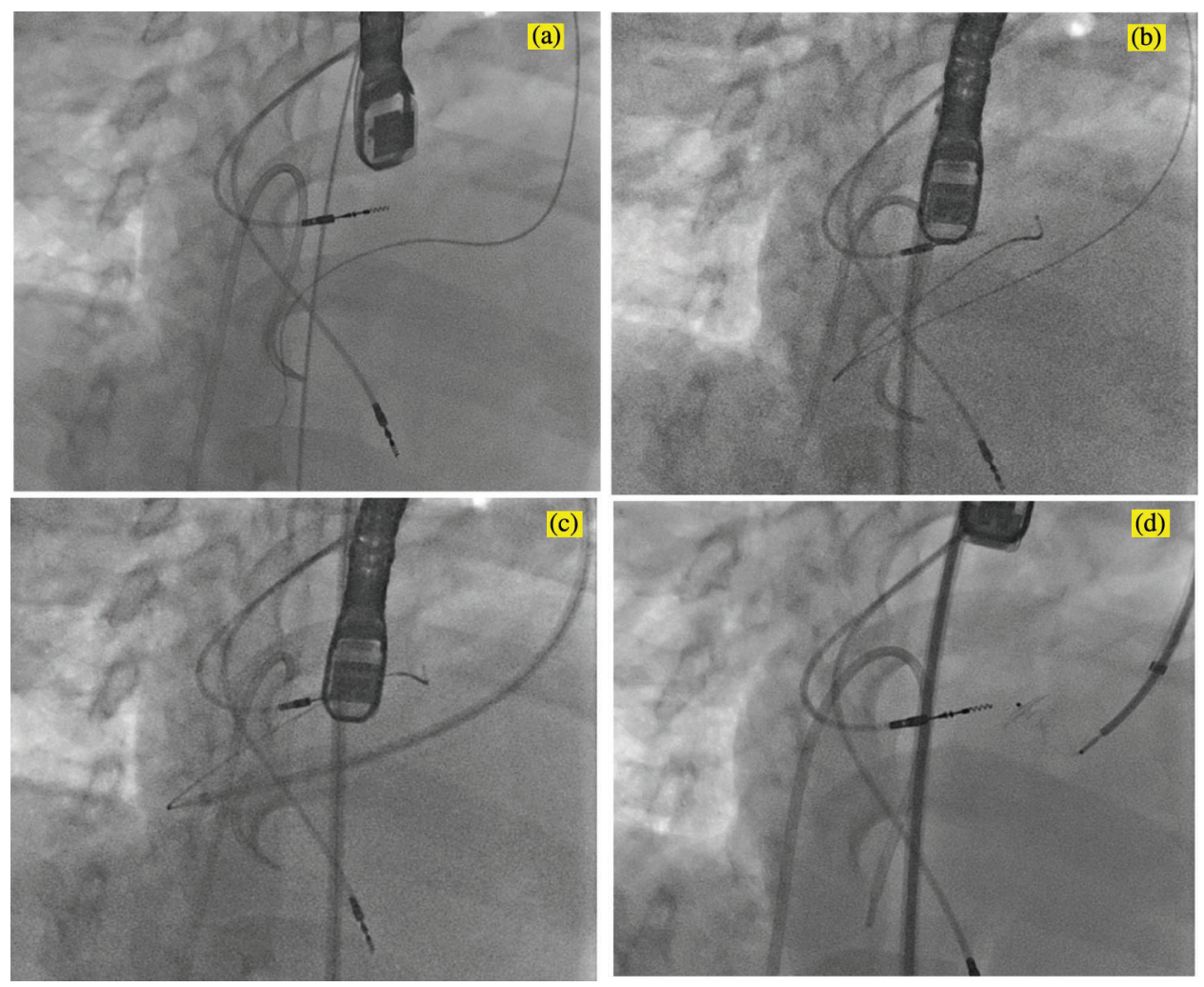

Figure 2: Fluoroscopic images in an RAO/Cranial projection demonstrating the steps of the VSD device closure. (a) An Amplatz Left-1 catheter was used to direct a 0.035 inch angle-tipped Terumo glide wire retrograde across the defect. The catheter was advanced cleanly across the defect without incident and used to form a redundant loop in the subpulmonary LV with a 0.035 inch Rosen guide wire (b). A TorqVue delivery system was advanced across the VSD and positioned in the LV retrograde over the Rosen wire (c). The device was then unsheathed (d); note that the RV disc is deformed due to the stiffness of the delivery cable despite no tension on the cable. LV, left ventricle; $R V$, right ventricle; VSD, ventricular septal defect

Angiography demonstrated the device in a good position with no residual shunt. TEE evaluation demonstrated no new aortic insufficiency and no change in the baseline mild tricuspid insufficiency. A trivial residual shunt was seen on TEE. During recovery and overnight observation, the patient demonstrated no change in baseline rhythm. He returned for uncomplicated percutaneous lead extraction of the malfunctioning ventricular lead and upgraded to a biventricular implantable cardioverterdefibrillator two months after his catheterization procedure. There was no residual shunt on intracardiac echocardiography (not pictured), and he returned home the next day. 

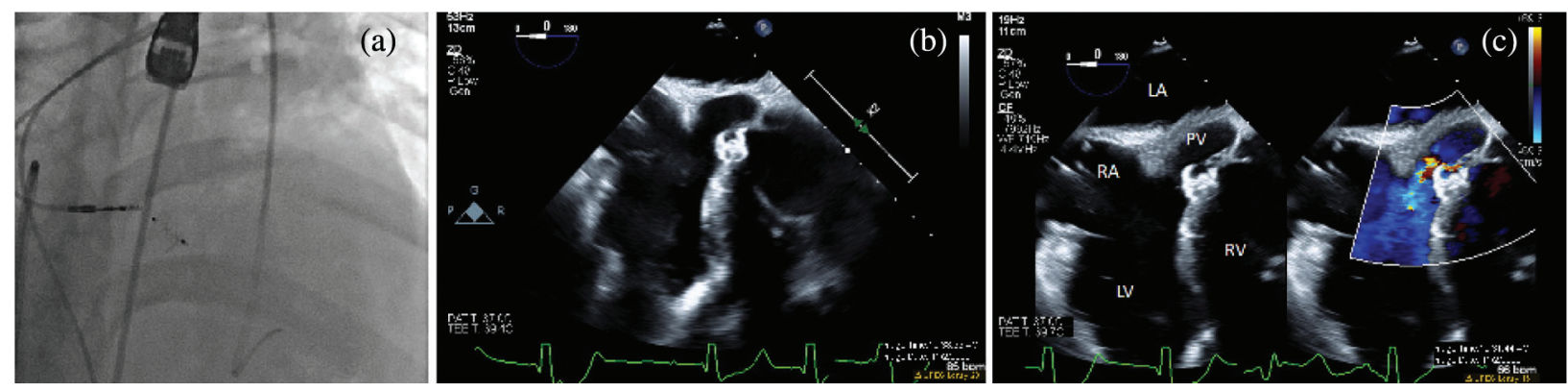

Figure 3: Post-intervention imaging. A fluoroscopic image in an $\mathrm{RAO} / \mathrm{Cranial}$ projection after device release (a). The device reorients and remains in a good position with no residual shunt on angiography. Transesophageal echocardiogram in a zero-degree view after device release in 2D (b) and with color compare (c). The device appears well seated across the ventricular septal defect, and color Doppler shows trivial residual flow around the superior edge of the device. LA, left atrium; LV, left ventricle; PV, pulmonary valve; $R A$, right atrium; $R V$, right ventricle

\section{Discussion}

To our knowledge, device closure of VSD has not been widely reported in L-TGA. This is in part due to its rarity; L-TGA accounts for only $0.6 \%$ of all congenital heart disease [1]. The coincidence of an anatomically appropriate VSD and an indication to address it without an indication for concomitant openheart surgery is even more unusual. In most circumstances, the risk of heart block poses a significant disincentive to transcatheter VSD closure. This case not only demonstrates the feasibility of transcatheter device closure of pmVSD in L-TGA but also illustrates several aspects of transcatheter VSD closure.

The technical challenges of transcatheter closure of pmVSD in D-looped ventricles have been described previously [3]. These challenges include: (1) obtaining a veno-arterial loop to allow for the antegrade deployment of the device (i.e., with the distal disc in the systemic ventricle), (2) avoiding heart block by relying on less rigid devices and/or seating the device in aneurysmal tricuspid valve tissue, and (3) optimal device sizing achieving effective closure and avoiding device impingement on the aortic and atrioventricular valves.

The pmVSD in this case (as is typical of L-TGA) was remote from the aortic valve and was in closer proximity to the pulmonary valve. Moreover, the trabeculated surface of the systemic right ventricle (RV) made cleanly crossing the VSD with our usual soft J-tipped wire impossible. Instead, after engaging the defect, we crossed with an angle-tipped glide wire. In addition, in this case, we felt that it was not desirable to pursue an antegrade deployment (wanting to secure the device first on the smooth-walled LV before deploying the RV disc). Crossing retrograde (i.e., from systemic to pulmonary ventricle) was easier because of the relatively small caliber of the TorqVue system required for an ADOII (relative to other similar devices) [4]. Deployment of the symmetric device also ensures that with a retrograde deployment, the device is secure. This has been shown in other series for small defects in D-looped ventricles [3-8].

To our knowledge, device closure of a VSD in L-TGA has been reported once previously. Mortera et al. [9] reported successful closure of a pmVSD in an 8-year-old with unrepaired L-TGA with a muscular VSD device. This device is rigid and likely to exert pressure on the conduction system, potentially posing a higher risk of heart block and valve tissue injury. In a patient with normal cardiac conduction and no pacemaker, we would hesitate before attempting device closure of the defect with this device. The ADOII device is more flexible [4-8] and seems to have less risk of causing conduction abnormalities than other devices [3-6]. 
Its use is limited by the narrow range of sizes that are commercially available (the largest diameter device is $6 \mathrm{~mm}$ in its central portion with $12 \mathrm{~mm}$ discs on either side).

In previous pmVSD closure cases by our team, real-time 3D echocardiography has been valuable in understanding whether there is a circumferential tricuspid valve aneurysm in which to seat the device and demonstrating the relationship of the VSD to its surrounding structures (especially the semi-lunar valves and tricuspid valve). 3D imaging is also especially useful for sizing defects. Similar to atrial septal defects, pmVSDs are not necessarily circular, making effective imaging with planar angiography or $2 \mathrm{D}$ echo challenging. Oversizing the device is not ideal both because of the proximity of the aortic valve and the potential increased risk of heart block with an oversized device, which is challenging on both planar angiography and 2D echocardiography.

\section{Conclusion}

This case demonstrates the feasibility of retrograde device closure of pmVSD in L-TGA. The coincidence of L-TGA with an appropriately sized pmVSD for device closure is rare, but this case is instructive about several aspects of transcatheter device closure for which the ADOII is particularly well-suited.

Author Contribution: The listed authors all made substantial contributions to the intellectual content of the article in terms of the conception, drafting, and revising of the work and the acquisition, analysis, and interpretation of the data. All authors take responsibility for the content of the manuscript.

Acknowledgement: The authors would like to acknowledge Doctors Sara Partington (Philadelphia Adult Congenital Heart Program), Stephanie Fuller (The Children's Hospital of Philadelphia (CHOP) and University of Pennsylvania Cardiothoracic Surgery), and Robert D. Schaller (University of Pennsylvania Section on Cardiac Electrophysiology) for participating in the involved discussions and complex care of this patient. They would also like to acknowledge Dr. Matthew Gillespie (CHOP Section on Interventional Cardiology) for his technical support and assistance during the case and Yan Wang (CHOP Echocardiography Laboratory) for contributing to the acquisition and processing of the TEE images.

Ethics Statement: Informed consent from the patient was obtained for the procedure. Our institutional review board (the Committees for the Protection of Human Subjects) deemed this retrospective case report exempt from the need for ethics board review and approval.

Funding Statement: The authors received no specific funding for this study.

Conflicts of Interest: The authors declare that they have no conflicts of interest to report regarding the present study.

\section{References}

1. Freedom, R. F. (2000). Congenitally corrected transposition of the great arteries. In: Moller, JH., Hoffman, JIE. (Eds.), Pediatric cardiovascular medicine, pp. 375-390. Philadelphia: Churchill Livingston.

2. Silka, M. J., Rice, M. J. (1991). Paradoxic embolism due to altered hemodynamic sequencing following transvenous pacing. Pacing and Clinical Electrophysiology, 14(4), 499-503. DOI 10.1111/j.1540-8159.1991.tb02820.x.

3. Morray, B. H. (2019). Ventricular septal defect closure devices, techniques, and outcomes. Interventional Cardiology Clinics, 8(1), 1-10. DOI 10.1016/j.iccl.2018.08.002.

4. Haddad, R. N., Daou, L., Saliba, Z. (2019). Device closure of perimembranous ventricular septal defect: Choosing between amplatzer occluders. Frontiers in Pediatrics, 7, 300. DOI 10.3389/fped.2019.00300. 
5. Bergmann, M., Germann, C. P., Nordmeyer, J., Peters, B., Berger, F. et al. (2021). Short- and long-term outcome after interventional VSD closure: A single-center experience in pediatric and adult patients. Pediatric Cardiology, 42(1), 78-88. DOI 10.1007/s00246-020-02456-2.

6. Wongwaitaweewong, K., Promphan, W., Roymanee, S., Prachasilchai, P. (2021). Effect of transcatheter closure by Amplatzer ${ }^{\mathrm{TM}}$ Duct Occluder II in patients with small ventricular septal defect. Cardiovascular Intervention and Therapeutics, 36(3), 375-383. DOI 10.1007/s12928-020-00677-z.

7. Kanaan, M., Ewert, P., Berger, F., Assa, S., Schubert, S. (2015). Follow-up of patients with interventional closure of ventricular septal defects with amplatzer duct occluder II. Pediatric Cardiology, 36(2), 379-385. DOI 10.1007/ s00246-014-1017-0.

8. Pamukcu, O., Narin, N., Baykan, A., Sunkak, S., Tasci, O. et al. (2017). Mid-term results of percutaneous ventricular septal defect closure with Amplatzer Duct Occluder-II in children. Cardiology in the Young, 27(9), 1726-1731. DOI 10.1017/S104795111700107X.

9. Mortera, C., Prada, F., Rissech, M., Bartrons, J., Mayol, J. et al. (2004). Percutaneous closure of ventricular septal defect with an amplatzer device. Revista Española de Cardiologia, 57(5), 466-471. DOI 10.1016/ S1885-5857(06)60177-1. 